\title{
Intermittent simulated hypoxia for pre-acclimatization
}

\author{
Markus Tannheimer
}

Received: 28 August 2009/Accepted: 25 September 2009/Published online: 15 October 2009

(C) Springer-Verlag 2009

Keywords Acclimatization $\cdot$ Hypoxia $\cdot$ Simulated altitude $\cdot$ Acute mountain sickness

Pre-acclimatization in hypoxic chambers is a new strategy to prevent high-altitude disorders during high-altitude exposure. As a matter of fact pre-acclimatization is requested in the wide range of tourism activity (source: http://www.hauser-exkursionen.de) instead of spending extra days on site in order to acclimatize following well established protocols [1, 2]. The assessment of this is discussed controversially and every physician familiar with high-altitude medicine has to decide if he is willing to support this. There is a wide range of high-altitude activity illustrated in the article by Küpper et al. [3]. Even if one initially thinks that pre-acclimatization is of no relevance for trekkers and mountaineers, actually, real altitude profiles especially exist in commercial offers (e.g., Kilimanjaro in 4 days; source: http://www.moja-travel.net). Nevertheless, acclimatization on site offers several advantages (testing material, team building, adaption to the environment, and nutrition...) that justify the additional time needed. In case of skiers in ski resorts at high altitude, e.g., Colorado, acclimatization is even more difficult because of missing capabilities and acceptance among the tourists. But in this special case medical support and a fast descent is always

\footnotetext{
M. Tannheimer $(\bowtie)$

Department of Visceral and Thoracic Surgery,

Armed Forces Hospital Ulm,

Oberer Eselsberg 40,

Ulm 89075, Germany

e-mail: markus.tannheimer@arcor.de
}

feasible in situations of high-altitude disorders, which makes un-acclimatized travel acceptable, even if skiing pleasure might be compromised. A proper acclimatization is of great importance for professional engagement at high altitude; however a step by step adaption process over several days seems to be more a hope than reality. In case of rescue and military [4] operations at high altitude, the exposition time and duration is often unknown and the subjects are not able to terminate the altitude stay by their own. Hypoxic chambers might be a solution to have staff prepared to leave for such missions.

In general, pre-acclimatization requires a defined amount of time, too. This opens the question if staying (and acclimatize) at the scene in the real environment makes more sense instead of spending this time in a hypobaric chamber. But recent investigations report beneficial effects from exposure times of only $1-4 \mathrm{~h}$ per day over $1-5$ weeks to simulated altitudes of about $4,000 \mathrm{~m}$ [5]. If these results are confirmed, a new dimension of acclimatization is at hand, for example, by simply spending the nights in a hypoxic chamber. And this would offer a new strategy in the above-mentioned situations. Up to now, these findings are discussed controversially, especially because there is no established protocol yet for acclimatization in interrupted hypoxia. Thus Küpper et al. (see above) report the current state of knowledge and they recommend a scheme for interrupted pre-acclimatization in a hypoxic chamber. Considering the findings above, the conclusion/statement seems conservative but shows the authors a careful approach. The step-by-step development of a reliable and safe procedure for acclimatization in a much shorter timeframe opens a field of important research. Hopefully, their article initiates the required investigation efforts with solid results presented in the near future. 


\section{References}

1. Bartsch P, Saltin B (2008) General introduction to altitude adaptation and mountain sickness. Scand J Med Sci Sports 18 (Suppl 1):1-10

2. Berghold F, Schaffert W, Pallasmann K (1991) Prerequisites and guidelines for acclimatization to great and extreme altitude in prevention of altitude sickness. Wien Med Wochenschr 141 (11):242-8

3. Küpper T, Schoeffl V (2009) Pre-acclimatization in hypoxic chambers for high altitude sojourns sleep breath (in press)

4. Muza SR (2007) Military applications of hypoxic training for highaltitude operations. Med Sci Sports Exerc 39(9):1625-31

5. Burtscher M, Brandstatter E, Gatterer H (2008) Preacclimatization in simulated altitudes. Sleep Breath 12:109-114 